\title{
Asociación entre variables reproductivas y anticuerpos anti Neospora caninum en bovinos lecheros de un municipio de Colombia
}

\section{Association between reproductive variables and anti Neospora caninum antibodies in dairy cattle herds from a Colombian municipality}

\author{
Martín Orlando Pulido Medellínª*, Adriana María Díaz Anayaa , Roy J osé Andrade Becerra
}

\begin{abstract}
RESUMEN
El objetivo fue determinar el porcentaje de vacas seropositivas a Neospora caninum y la identificación de las variables reproductivas que se encuentran relacionadas con la presencia de anticuerpos contra este protozoo. Se tomaron 1,000 muestras de sangre a hembras bovinas del municipio de Sotaquirá, Boyacá, las cuales fueron evaluadas por medio de ELISA indirecta. Se determinó una seroprevalencia de Neospora caninum del $45 \%$; las variables reproductivas asociadas con la positividad de $N$. caninum fueron: la presencia de distocia en el parto, el intervalo de edad (3-4 años) y la raza de los bovinos (Holstein), mientras que las variables de presencia de aborto, inseminación artificial, muerte embrionaria, repetición de celo, presentación de terneros débiles al nacer y monta directa con toro, no presentaron relación con la positividad de los bovinos. Los resultados de este estudio muestran la presencia de anticuerpos contra Neospora caninum en animales de producción lechera, siendo esta prevalencia encontrada una muestra del riesgo potencial que representa esta enfermedad sobre el estado reproductivo dentro de los hatos.
\end{abstract}

PALABRAS CLAVE: Anticuerpos, Elisa indirecta, Neospora caninum, Prevalencia, Distocia, Bovinos.

\begin{abstract}
The aim of this study was to determine the percentage of seropositive cows to Neospora caninum and the identification of reproductive variables related to the presence of antibodies against this protozoan. One thousand $(1,000)$ blood samples were collected of cows from Sotaquirá, Boyacá. The samples were runned by ELISA indirect technique. A Neospora caninum seroprevalence antibodies of $\mathbf{4 5} \%$ was determined; the reproductive variables associated were: presence of dystocia, age (3- 4 yr) and breed (Holstein), about the abortion, artificial insemination, embryonic death, return to estrus, weak calves at birth and natural breeding were not associated with the presence of specific antibodies against the protoozoo. The results of this study showed a presence of specific antibodies against to Neospora caninum in dairy cows, suggesting that the Neosporosis may have a negative impact on reproductive status of the herds.
\end{abstract}

KEY WORDS: Antibodies, Indirect ELISA, Neospora caninum, Prevalence, Dystocia, Cattle.

Recibido el 18 de marzo de 2016. Aceptado el 3 de junio de 2016.

a GIDIMEVETZ, Programa de Medicina Veterinaria y Zootecnia, Universidad Pedagógica y Tecnológica de Colombia. Tunja, Colombia.

* Autor de correspondencia: mopm1@hotmail.com. 


\section{INTRODUCCIÓN}

Neospora caninum es un parásito protozoo intracelular obligado, perteneciente al phylum Apicomplexa, familia Sarcocystidae, morfológicamente similar a Toxoplasma gondii, y está relacionado taxonómicamente a otros protozoos formadores de quistes como Hammondia heydornie Isospora bigemina. Mediante microscopía electrónica se reconocen organelas características de ese phylum como por ejemplo, micronemas, roptrias y gránulos densos ${ }^{(1)}$. Neospora caninum es uno de los parásitos más importantes que causan abortos bovinos alrededor del mundo, pero a pesar del impacto económico que ocasiona se conoce poco sobre este parásito(2).

La transmisión de $N$. caninum se realiza mediante dos importantes formas, la transmisión vertical que puede ocurrir a través del ciclo exógeno y endógeno, y la transmisión horizontal, la cual solo ocurre por medio del ciclo exógeno, donde el bovino debe ingerir alimento o agua contaminados con ooquistes esporulados del parásito, que excreta el perro, principal portador definitivo de $N$. caninum ${ }^{(3)}$. Varios estudios demuestran que la infección puede transmitirse verticalmente de la madre al ternero, siendo esta vía epidemiológicamente muy importante, lo cual explicaría el comportamiento enzoótico de la enfermedad en los hatos ganaderos ${ }^{(4)}$.

Diversos estudios sobre neosporosis bovina en Colombia, han demostrado la presencia del parásito en los hatos lecheros, relacionando éste, posiblemente con la presencia de abortos, causando grandes pérdidas económicas en las explotaciones de bovinos productores de leche, así como aumento de los costos indirectos asociados al diagnóstico veterinario, repetición de la inseminación, pérdidas de producción láctea y los costos de reemplazo por descarte de $\operatorname{vacas}^{(5,6)}$. Los abortos frecuentes se convierten en el principal problema de las ganaderías lecheras presentándose de forma esporádica, endémica o epidémica con origen infeccioso o no infeccioso, por lo que resulta difícil determinar el agente causal(7). Dentro de estos agentes se encuentra Neospora caninum, un protozoo intracelular obligado que ha estado relacionado con la presencia de abortos, tanto en países desarrollados como países latinoamericanos incluyendo México, Brasil, Chile, Paraguay y Argentina ${ }^{(8)}$.

Dentro de los problemas que se pueden presentar en un hato lechero infectado con $N$. caninum están muerte fetal temprana con repetición de celo, incremento del intervalo entre partos, concepción o infertilidad, aborto en el tercio medio de la gestación, muerte perinatal o neonatal o incremento en el deshecho de vacas. Las vacas infectadas tienen más probabilidad de ser descartadas por bajo desempeño reproductivo( ${ }^{(9)}$.

El departamento de Boyacá posee varios municipios con una importante producción láctea, en los cuales se presentan numerosos abortos, motivo por el cual, se hace necesario determinar la prevalencia de anticuerpos contra este protozoo en un municipio de gran importancia lechera, con el fin de identificar las variables reproductivas que se encuentran relacionadas con la presencia de $N$. caninum

\section{MATERIAL Y MÉTODOS}

\section{Área de estudio}

El estudio se llevó a cabo en el municipio de Sotaquirá, que está situado en el Departamento de Boyacá, Provincia del Centro, en las coordenadas 5o 46 ' 52" N, 73o 15' O del meridiano de Greenwich y $0^{\circ} 49^{\prime} 25^{\prime \prime}$ de Longitud con relación al meridiano de Bogotá. Limita por el este con el municipio de Tuta, por el norte con el municipio de Paipa, por el oeste con el municipio de Gambita y por el sur con el municipio de Combita. Su extensión total es de $288.65 \mathrm{~km}^{2}$, el área urbana abarca $0.1 \mathrm{~km}^{2}$, el área rural consta de $288.55 \mathrm{~km}^{2}$. Presenta temperatura anual promedio de $14{ }^{\circ} \mathrm{C}$, con 2,860 msnm. La explotación ganadera ocupa el $37 \%$ del área agropecuaria del municipio, equivalente a 10,582 ha aproximadamente, de las cuales 7,150 contienen pastos mejorados y 3,432 ha cultivadas con pastos naturales, concluyendo que el $67.5 \%$ de la actividad se desarrolla a través de una explotación intensiva y el $32.5 \%$ extensiva; para el 2014 se contaba con una población de 13,464 cabezas bovinas ${ }^{(10)}$. 


\section{Animales y tamaño de la muestra}

Se calculó el tamaño de muestra para los bovinos mediante la ecuación descrita por Pourhoseingholi et $a^{(11)}$, donde: $\mathrm{N}=13,464$ bovinos, $\mathrm{Za}=1.96$ a un nivel de confianza del $95 \%, \mathrm{e}=5 \%$, margen de error admitido, $\mathrm{P}=50 \%$, prevalencia esperada, esto debido a que, en Sotaquirá no se conocen estudios relacionados de seroprevalencia de $N$. caninum en bovinos. El tamaño de muestra calculada fue de 374 animales, sin embargo se recolectaron aleatoriamente 1,000 muestras procedentes de 67 hatos ubicados en Sotaquirá, realizando un muestreo mínimo de animales por explotaciones de bovinos productores de leche.

Mediante la aplicación de una encuesta al momento de recolección de las muestras, se tomaron datos de raza, edad y estado reproductivo de los animales. Así mismo, se obtuvieron de los registros de las fincas los datos de variables reproductivas, como gestante, no gestante, abortos, distocias, inseminación artificial, muerte embrionaria, repetición de celos y monta natural, con el fin de identificar las variables relacionadas con la presentación de $N$. caninum.

En cuanto a la edad, se dividieron en tres grupos de la siguiente manera: animales 2-3 años, 3-4 años y >4 años.

Las muestras se obtuvieron a partir de venopunción coccígea utilizando tubos Vacutainer,

Figura 1. Presencia de anticuerpos contra Neospora caninumen vacas Holstein

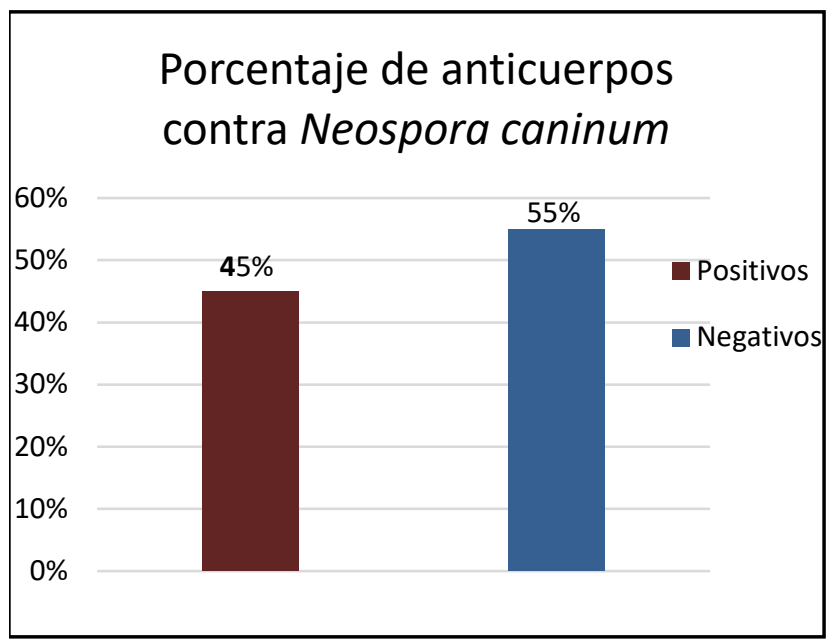

que por ser un sistema cerrado presta mayor garantía en cuanto asepsia y presenvación de las muestras. El tubo que contenía la sangre sin anticoagulante se centrifugó a 1,500 rpm durante 10 min para separar el suero. Luego con una pipeta Pasteur se separó el suero y se transfirió a un tubo de almacenamiento para la realización de las pruebas ${ }^{(12)}$.

Las muestras se procesaron bajo la técnica de ELISA indirecta en el Laboratorio de parasitología de la Universidad Pedagógica y Tecnológica de Colombia, utilizando el kit comercial para Neospora caninum Ingezim R.12.NC.K1 (Ingenasa S.A) y se siguió el protocolo del fabricante. Este kit comercial refiere una sensibilidad $99.4 \%$ y especificidad del $100 \%$.

El estudio fue descriptivo de corte transversal, el diseño del muestreo fue aleatorio simple, observando la frecuencia con que se presentaron anticuerpos contra el protozoo. Para el análisis del estudio, se utilizó el paquete estadístico SPSS 18.

\section{RESULTADOS}

Después de haber analizado las muestras de suero bovino se encontró una seropositividad del $45 \%(450 / 1000)$ de anticuerpos contra de $N$. caninum (Figura 1 ).

Se realizó la prueba de Ji cuadrada de asociación-independencia para determinar si existía relación entre la presencia de anticuerpos contra $N$. caninum y las diferentes variables reproductivas (Cuadro 1). No se encontró relación significativa

Cuadro 1. Parámetros estadísticos con respecto a las variables reproductivas asociadas a la presencia de anticuerpos contra $N$. caninum

\begin{tabular}{lcc}
\hline Variables reproductivas & CHI $\mathbf{2}^{*}$ & OR $^{* *}$ \\
\hline Aborto & 0.033 & 1.03 \\
Distocias & 4.241 & 1.33 \\
Inseminación artificial & 1.872 & 0.83 \\
Muerte embrionaria & 2.362 & 1.32 \\
Repetición de celo & 2.925 & 1.25 \\
Terneros débiles al nacer & 1.391 & 1.26 \\
Monta directa con toro & 2.801 & 1.26 \\
\hline
\end{tabular}

Intervalo de confianza de 95\%.

* Ji cuadrada. 
entre $N$. canimun y la presencia de aborto en las hembras ( $P=0.856$ ) (Figura 2 ).

Se encontró una relación significativa entre $N$. caninum y la presentación de distocias $(P=0.039)$, así mismo, se encontró diferencia para los intervalos de edad establecidos ( $P=0.005$ ) (Figura 3).

No se encontró relación significativa entre la presencia de anticuerpos contra $N$. caninum e inseminación artificial $(P=0.171)$. En animales en los que se usa inseminación artificial la presencia de anticuerpos fue del $42.9 \%$, mientras en los animales que no usaron inseminación artificial fue del $47.4 \%$. De igual forma, no se encontró relación significativa entre $N$. caninum y muerte embrionaria $(P=0.124)$.

Para la variable raza, la prueba indicó que si hay por lo menos un valor observado que es diferente al esperado $(P=0.001)$. Se presentó un $14.8 \%$ de bovinos raza Holstein con presencia de anticuerpos contra $N$. caninum y un $12.2 \%$ de bovinos raza Ayrshare, con un menor número de casos de las razas Normando (10\%) y J ersey (8\%) (Figura 4).

No se encontró asociación significativa entre las hembras que presentaron anticuerpos contra a $N$. caninum y repetición de celo $(P=0.087)$, presentación de terneros débiles al nacer $(P=0.856)$, ni monta directa con toro $(P=0.094)$.

Figura 2. Presencia de anticuerpos contra Neospora caninum y su relación con la presencia de aborto en hatos lecheros

\section{Porcentaje de anticuerpos contra N.caninum según aborto}

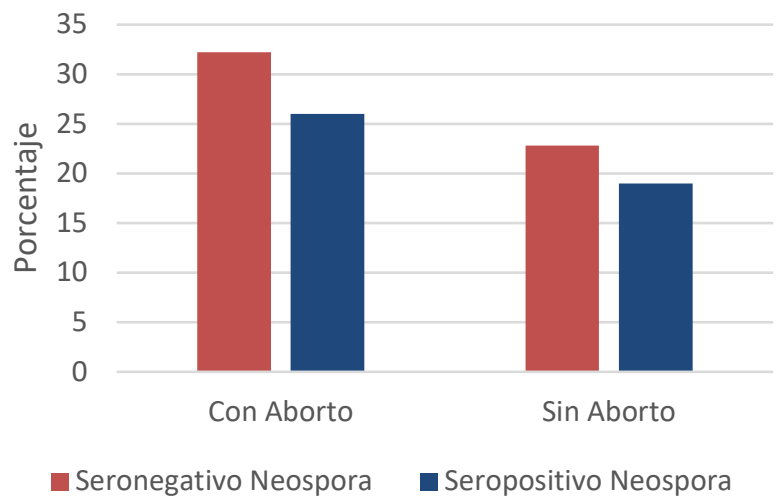

\section{DISCUSIÓN}

Existen numerosos reportes sobre infecciones por Neospora caninum en la especie bovina, en la mayoría de los casos utilizando medios de diagnóstico serológicos, en los cuales se reporta una prevalencia a nivel mundial que oscila desde 2.5 al

Figura 3. Presencia de anticuerpos contra Neospora caninum y su relación con la edad de los bovinos

\section{Porcentaje de anticuerpos contra Neospora caninum según edad}

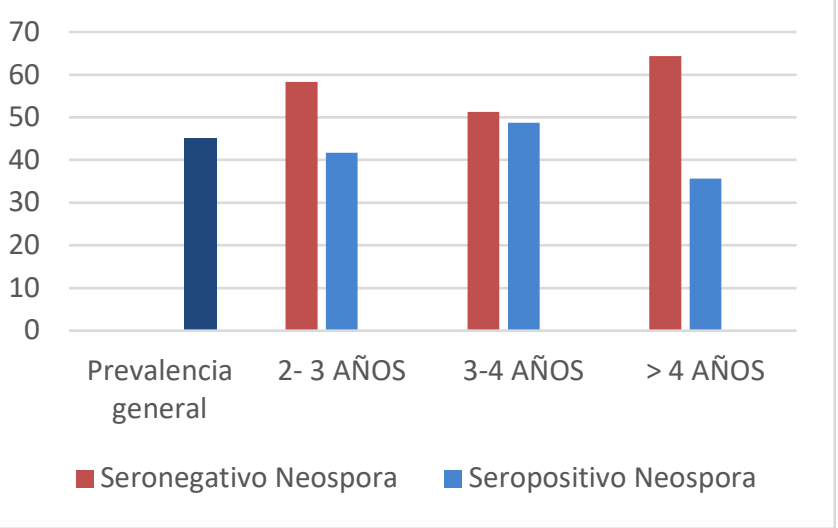

Figura 4. Presencia de anticuerpos contra Neospora caninum y su relación con la raza de los bovinos del municipio de Sotaquirá, Boyacá

\section{Porcentaje de anticuerpos contra N. caninum segun raza}

- Seronegativo Neospora Seropositivo Neospora

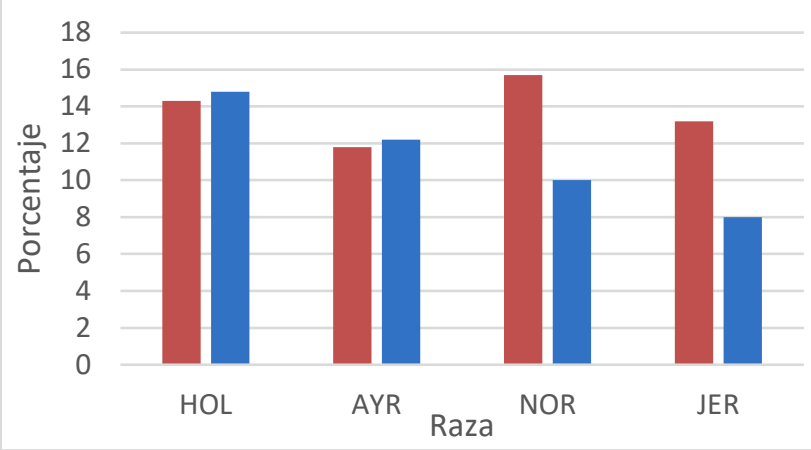


$80.0 \%(4,6,13-16)$. A pesar de esto, en Colombia son escasos los reportes acerca de la presencia del parásito en ganadería de leche y su relación con variables reproductivas en los hatos.

La presencia de anticuerpos encontrada en esta investigación fue del $45 \%$, cercana a la encontrada en la provincia de Sugamuxi en Boyacá, de 57.7 $\%{ }^{(17)}$. Este resultado es superior al encontrado por Motta et a ${ }^{(18)}$ quienes realizaron un estudio en el departamento del Caquetá, en hatos bovinos y bubalinos, obteniendo una prevalencia de $12.5 \%$ y $45.4 \%$ respectivamente. La prevalencia del presente estudio sigue siendo mayor a las obtenidas en Córdoba( ${ }^{(6)}$ cuya prevalencia fue de $10.2 \%$; de $34.6 \%{ }^{(5)}$, y baja respecto al estudio realizado por Peña et $a{ }^{19)}$, en el Valle del Cesar, donde se encontró una seroprevalencia del $68 \%$.

Trabajos realizados acerca de la presencia de anticuerpos contra a $N$. caninum mediante diagnóstico serológico en otros países, reportan datos como es el caso de Venezuela con $20.6 \%{ }^{(20)}$, Ecuador con $19.5 \%{ }^{(21)}$, México con prevalencias entre 30 y $44 \%{ }^{(4,22)}$, siendo esta última cercana a la obtenida en este estudio.

A pesar de que la neosporosis está relacionada estrechamente con fallas reproductivas, los resultados encontrados no mostraron una asociación entre $N$. caninumy aborto; sin embargo, el $19 \%$ de las hembras bovinas fueron positivas a anticuerpos contra $N$. caninum donde según los registros habían presentado aborto en algún momento del año; las hembras infectadas con neospora tienen aproximadamente 1.03 veces más probabilidad de sufrir aborto que las hembras sin la presencia de anticuerpos. Lo anterior, indica que este parásito puede infectar los bovinos pero no ser la causa directa del aborto, teniendo en cuenta que existen otros agentes etiológicos que también producen abortos $^{(4)}$.

Se encontró que el $23 \%$ de las hembras no presentaron anticuerpos contra el parásito pero registraron abortos, lo cual sugiere que dichos animales no estarían libres de la infección con $N$. caninum, ya que vacas negativas han abortado fetos infectados con el parásito, detectados mediante $P C R^{(23)}$; también puede ser el resultado de animales no expuestos al parásito o animales expuestos al parásito en un tiempo pasado, pero sin anticuerpos. Sin embargo, no se puede establecer una clara relación entre la infección y el aborto únicamente con una prueba serológica, ya que ésta solo puede revelar la exposición al agente sin diferenciar infecciones viejas o recientes ${ }^{(24)}$.

No obstante, sí se encontró una asociación entre animales positivos y la presentación de distocia en un $16.6 \%$ de las hembras bovinas de Sotaquirá, resultado consistente con un estudio realizado en Canadá, donde se diagnosticó distocia en un $22.5 \%$. Este diagnóstico fue hecho a partir de historia clínica y lesiones tales como congestión y edema de cuello y cabeza, manchas de meconio en el cuerpo, contusiones y fracturas o costillas magulladas en terneros ${ }^{(25)}$.

Un estudio comparativo de la seroprevalencia de $N$. caninum realizado entre cuatro países: Suecia, Alemania, España y Países Bajos, encontró una prevalencia del parásito en ganado de leche del 16, 49, 63 y $76 \%$ respectivamente ${ }^{(26)}$; estos estudios demostraron que la mayor prevalencia del parásito se registra en ganado de leche, existiendo diferencias regionales en la distribución del $N$. caninum según variables climáticas, manejo de los sistemas de producción y cantidad de perros presentes en los hatos, siendo solo algunas de las distintas posibilidades de transmisión de la enfermedad ${ }^{(27)}$.

Al analizar los datos referentes a la edad en animales positivos a $N$. caninumse puede evidenciar que en todos los grupos etarios analizados hay presencia del parásito; sin embargo, la edad con mayor número de animales infectados corresponde a las hembras de 3-4 años con un $29.8 \%$, sin dejar de ser importante la prevalencia encontrada en el grupo >4 años, esto debido a que el riesgo de ser seropositivo incrementa con la edad según Bartels et $a{ }^{(26)}$, indicando la alta probabilidad de transmisión horizontal. Lo anterior puede deberse a la exposición postnatal de los bovinos con fuentes contaminadas por ooquistes esporulados del parásito(20). Al respecto, algunas investigaciones indican que la edad no parece comportarse como un factor de riesgo asociado a la neosporosis ${ }^{(28,29,30)}$, en cuyo caso se sugiere que las infecciones son 
predominantemente transmitidas por vía transplacentaria, pudiendo aumentar de esta manera la proporción de animales infectados dentro de los hatos(31).

No se encontró una asociación significativa ente las hembras positivas a $N$. caninumy la presentación de muerte embrionaria; esto se debe posiblemente a que las causas de aborto bovino y muertes embrionarias son difíciles de determinar por diversas razones, ya que cualquier perturbación puede provocar aborto y alteración en la fisiología normal de la gestación, por lo tanto, otras causas como agentes infecciosos, toxinas, desequilibrio hormonal, vacunación, nutrición deficiente, alteraciones cromosómicas y factores físicos, pueden producir aborto y muerte embrionaria(24).

La prevalencia en cuanto a la raza bovina depende del país, región y tipo de prueba serológica utilizada, llegándose a determinar en algunos rebaños de vacas lecheras sobre el $87 \%$ de seropositividad ${ }^{(32)}$. Durante la investigación se evidenció una mayor presentación del parásito en hembras de raza Holstein, con $14.8 \%$. Los resultados coinciden parcialmente con los obtenidos por investigadores brasileños, quienes encontraron que hembras bovinas Holstein tienen 2.13 veces más oportunidad de ser seropositivas a $N$. caninum que las de razas cebú o mestizas ${ }^{(16,33)}$.

En condiciones de repetición de celo no se encontró una relación significativa en hembras positivas a $N$. caninum, esto coincide con un trabajo realizado en Brasil ${ }^{(34)}$. Diferente de otro estudio en el que se demostró que las vacas seropositivas requieren un mayor número de inseminaciones por concepción que las vacas seronegativas ${ }^{(35)}$.

Durante el estudio no se encontró relación entre terneros que nacieron débiles y hembras seropositivas al parásito. Sin embargo, se conoce que tenernos infectados con $N$. caninum pueden nacer con signos neurológicos, peso bajo e incapaces de levantarse. El examen neurológico puede revelar ataxia, disminución del reflejo patelar y pérdida de la propiocepción ${ }^{(36)}$.

En cuanto al método de reproducción por monta natural o inseminación artificial, no se encontró una relación significativa entre estos y la presentación del parásito; sin embargo, DNA de $N$. caninum se ha encontrado en el esperma de los toros expuestos de forma natural, y aunque teóricamente es posible, la transmisión venérea a través del esperma contaminado se considera poco probable $^{(1)}$.

Entre las posibles causas que contribuyen a la prevalencia del $45 \%$, se puede destacar que en la mayoría de las explotaciones bovinas donde se muestrearon los animales, los protocolos sanitarios pueden llegar a ser insuficientes, pues sólo se vacuna contra las enfermedades consideradas de notificación obligatoria a nivel nacional (brucelosis bovina y fiebre aftosa); tampoco hay establecidos planes de desparasitación constantes, que permitan mantener el control de parásitos internos y externos. La alimentación es por pastoreo, lo que hace posible que existan ooquistes esporulados depositados en el pasto hace mucho tiempo por otros animales infectados.

Otro factor determinante para que se encontrara esta presencia de anticuerpos contra $N$. caninumen el municipio de Sotaquirá, tiene que ver con la presencia de caninos en la mayoría de las fincas, donde es normal que estos deambulen por los potreros donde pastan las vacas, e incluso es muy común que estos estén presentes a la hora del ordeño, resaltando que estos animales son la fuente primaria de infección de los bovinos, representando un factor de riesgo muy importante dado que los caninos expulsan los ooquistes del parásito en sus heces, facilitando la transmisión horizontal de la neosporosis ${ }^{(37)}$.

\section{CONCLUSIONES E IMPLICACIONES}

La seropositividad de anticuerpos contra Neospora caninum encontrada en vacas lecheras del municipio de Sotaquirá, Boyacá (45 \%), fue elevada si se tiene en cuenta los datos de seroprevalencia que reporta la literatura en otras regiones del país. Las variables asociadas con la seropositividad fueron la presencia de distocia en el parto, el intervalo de edad (3-4 años) y la raza de los bovinos (Holstein), mientras que las variables de presencia de aborto, inseminación artificial, muerte embrionaria, repetición de celo, presentación de terneros débiles 
al nacer y monta directa con toro, no presentaron relación con la seropositividad de los bovinos.

\section{AGRADECIMIENTOS}

A Laboratorios Vecol por el financiamiento de este proyecto, de igual forma a Copoica y Zoolab por su asesoría científica.

\section{LITERATURA CITADA}

1. Dubey JP, Schares G, Ortega-Mora L. Epidemiology and control of Neosporosis and Neospora caninum. Clin Microbiol Rev 2007; 20(2):323-367.

2. Salehi N, Gottstein B, Haddadzadeh HR. Genetic diversity of bovine Neospora caninum determined by microsatellite markers. Parasitol Int 2015;64(5):357-361.

3. Santana O, Ramos M, Cruz, C, Castellano C, Medina L, Quezada D. Neospora caninum. Detección de ADN en sangre durante la primera gestación de vaquillas infectadas naturalmente. Vet Mex 2010;41(2):131-137.

4. Obando C, Bracamonte M, Montoya A, Cadenas V. Neospora caninum en un rebaño lechero y su asociación con el aborto. Rev Cien FCV-LUZ 2010;20(3):235-239.

5. Oviedo T, Betancur C, Mestra A, González M, Reza L, Calonge K. Estudio serológico sobre neosporosis en bovinos con problemas reproductivos en Montería, Córdoba, Colombia. Rev MVZ Córdoba 2007; 12(1):929-933.

6. López G, Restrepo B, Restrepo M, Lotero M, Murillo V, Chica A, Cano J, Giraldo J. Estudio para evidenciar la presencia de Neospora caninum en bovinos de la hacienda San Pedro en el municipio de Fredonia. Rev MVZ CES 2007:2(1): 7-20.

7. Neverauskas CE, Nasir A, Reichel MP. Prevalence and distribution of Neospora caninum in water buffalo (Bubalus bubalis) and cattle in the Northern territory of Australia. Parasitol Int 2015;64(5):392396. doi: 10.1016/j.parint.2015.05.009.

8. Lozada E. Determinación de la presencia de anticuerpos a Neospora caninum en hatos lecheros de la sierra norte del Ecuador, por prueba inmunoenzimatica [tesis doctorado]. Universidad Central del Ecuador. 2004.

9. Fort M, Edelsten M, Maley S, Innes E. Seroepidemiological study of Neospora caninum in beef and dairy cattle in La Pampa, Argentina. Acta Parasitol 2015;60(2):275-282. doi: 10.1515/ap-2015-0039.

10. Alcaldía de Sotaquirá. Información general. Boyacá, Colombia. http://www.sotaquira-boyaca.gov.co/index.shtml\#2. 2014.

11. Pourhoseingholi MA, Vahedi $M$, Rahimzadeh $M$. Sample size calculation in medical studies. Gastroenterol Hepatol Bed Bench 2013;6:14-17.

12. Muñoz M, Morón C. INS: Manual de procedimientos de laboratorio en técnicas básicas de hematología. ISBN 9972- 857 - 26 - 3 (O.C.). http://bvs. minsa.gob.pe/local/INS/845 MS-INS-NT40.pdf. 2005. Consultada 12 Mar, 2016.

13. Kowalczyk SJ , Czopowicz M, Weber CN, Müller E, Witkowski L, Kaba J. Herd-level seroprevalence of Neospora caninuminfection in dairy cattle in central and northeastern Poland. Acta Parasitol 2016;61(1):63-75. doi: 10.1515/ap-2016-0006.

14. Sun WW, Meng QF, Cong W, Shan XF, Wang CF, Qian AD. Herd-level prevalence and associated risk factors for Toxoplasma gondii, Neospora caninum, Chlamydia abortus and bovine viral diarrhoea virus in commercial dairy and beef cattle in eastern, northern and northeastern China. Parasitol Res 2015;114(11):4211-4218. doi: 10.1007/s00436-015-4655-0.

15. Nasir A, Lanyon S, Schares G, Anderson M, Reichel M. Seroprevalence of Neospora caninum and Besnoitia besnoiti in South Australian beef and dairy cattle. Vet Parasitol 2012; 186:480-485.

16. Escalona J, García F, Mosquera O, Vargas F, Corro A. Factores de riesgo asociados a la prevalencia de Neosporosis bovina en el municipio Bolívar del estado Yaracuy, Venezuela. Zoot Trop 2010;28(2):201-211.

17. Pulido Medellín M, Díaz Anaya A, García D, Andrade Becerra D. Determinación de anticuerpos anti Neospora caninum en vacas de la provincia de Sugamuxi, Colombia. Rev Mex Cienc Pecu 2013;4(4):501-506.

18. Motta GJ L, Clavijo HJA, Waltero GI, Abeledo MA. Prevalencia de anticuerpos a Brucella abortus, Leptospira sp. y Neospora caninum en hatos bovinos y bubalinos en el Departamento de Caquetá, Colombia. Rev Salud Anim 2014;36(2):80-89.

19. Peña L, Rubio DE, Mojica CP, Falquez JL, Avendaño KE. Estudio serológico de DVB, IBR y Neospora en bovinos de la Microrregión del Valle del Cesar. Rev Colomb Microbiol Trop 2012;2(2):35-40.

20. Fernández FJG, García F. Diagnóstico serológico de Neosporosis bovina en fincas de la región de Tucacas, estado Falcón. Venezuela Zootecnia Trop 2013;3(14):291-298

21. Burgasi CEG. Determinación de la prevalencia de las principales enfermedades infecciosas y endoparasitarias en hatos lecheros de pequeños productores, en las comunidades de Taxojaló y Guantualó, del cantón Sigchos provincia de Cotopaxi [tesis de pregado]. Escuela Superior Politécnica de Chimborazo. Riobamba Ecuador. 2014.

22. Sierra RC, Medina EL, Parra RM, Garcia VZ, Cruz VC. Factores de riesgo asociados a la seroprevalencia de anticuerpos a Neospora caninum en ganado lechero de Aguascalientes, México. Rev Mex Cienc Pecu 2001;2(1): 15-24.

23. Sager $H$, Fischer $K$, Furrer $M$, Strasser A, Waldvogel $P$, Boerlin $L$, Audigé B, Gottstein. A Swiss case-control study to assess Neospora caninumassociated bovine abortions by PCR, histopathology and serology. Vet Parasitol 2003; 102:1-15.

24. Lucchese L, Benkirane A, Hakimi I, El Idrissi A, Natale A Seroprevalence study of the main causes of abortion in dairy cattle in Morocco. Vet Ital 2016;52(1):3-19. doi: 10.12834/Vetlt.388.1813.1.

25. Khodakaram-Tafti A, I kede BO. A retrospective study of sporadic bovine abortions, stillbirths, and neonatal abnormalities in Atlantic Canada, from 1990 to 2001. Can Vet J 2005;46(7):635-637.

26. Bartels C, Arnaiz J, Ruiz A, Björkman C, Frössling J, Von Blumröder $D$, et al. Supranational comparison of Neospora caninum seroprevalences in cattle in Germany, the Netherlands, Spain and Sweden. Vet Parasitol 2006;137:17-27.

27. Frossling J, Nodtvedt A, Lindberg A, Bjorkman C. Spatial analysisof Neospora caninum distribution in dairy cattle from Sweden. Geospat Health 2008; 3:39-45. 
28. Fernández JG. Seropositividad de la neosporosis bovina en fincas ganaderas de la Región de Tucacas, estado Falcón. Trabajo de Grado. FCV-UCV, Maracay, Venezuela. 2004.

29. Bañales P, Fernández L, Repiso MV, Gil A, Dargatz DA, Osawa T. A nationwide survey on seroprevalence of Neospora caninum infection in beef cattle in Uruguay. Vet Parasitol 2006; 139:15-20.

30. Suárez C, Maldonado J. Seropositividad a Neospora caninum en unidades de producción bovina del estado Lara, Venezuela. Zoot Trop 2012; 30(1):35-41.

31. Frössling J, Uggla A, Björkman C. Prevalence and transmission of Neospora caninum within infected Swedish dairy herds. Vet Parasitol 2005; 128:209-218.

32. Dubey JP. Review of Neospora caninumand neosporosis in animals. Korean J Parasitol 2003; 1(1):1-16.

33. Guimarães J S, Souza SLP, Bergamaschi DP, Gennari SM. Prevalence of Neospora caninum antibodies and factors associated with their presence in dairy cattle of the north of Paraná state, Brazil. Vet Parasitol 2004; 124:1-8.

34. Ferreira-De-Oliveira V, Álvarez-Garcia G, Ortega-Mora L, FerreiraBorges L, Caetano Da Silva A. Abortions in bovines and Neospora caninum transmission in an embryo transfer center. Vet Parasitol 2010; 173:206-210.

35. Hall CA, Reichel MP, Ellis JT. Neospora abortion in dairy cattle: diagnosis, mode of transmission and control. Vet Parasitol 2005; 128:231-241.

36. Mazuz ML, Fish L, Reznikov D, Wolkomirsky R, Leibovitz B, Savitzky $\mathrm{I}$, et al. Neosporosis in naturally infected pregnant dairy cattle. Vet Parasitol 2014;205(1-2):85-91. doi: 10.1016/j.vetpar.2014.06.009.

37. Portocarrero $\mathrm{C}$, Pinedo R, Falcón N, Chávez A. Factores de riesgo asociados a la seroprevalencia de Neospora caninum en bovinos naturalmente Infectados en la Ceja de Selva de Oxapampa, Perú. Rev Inv Vet Perú 2015;26(1):119-126. 